\title{
Breakthroughs and Issues in HPV Genital Infections in relation to Cervical Cancer
}

\author{
Farhat Hussain ${ }^{1}$, Khairun Nahar ${ }^{2}$, Kaniz Fatema ${ }^{3}$, Mahbuba Khan ${ }^{4}$
}

\begin{abstract}
The field of cervical cancer prevention is rapidly evolving because of identification of the cause of disease. Human Papilloma Virus (HPV) has been recognized as a necessary cause of cervical cancer, and the association applies equally to both squamous cell and adenocarcinoma and is consistent worldwide. HPV 16 and 18 together attribute $70 \%$ of world's cervical cancer. HPV is highly prevalent among sexually active women and traceable in its natural history with technology that can detect HPV DMA at all stages of infection and neoplastic process. HPV DMA testing represents the scientifically obvious next step after Pap test for secondary prevention. A clinically validated and FDA approved HPV test has proven a substantial gain in sensitivity with a limited loss in speciality as compared to the standard Pap smear. We are now in the fortunate position of having two highly promising HPV vaccines in the pipeline. These vaccines today represent new hope for protection against cervical cancer, pre-cancerous cervical lesions and other HPV related condition for the current generation of adolescents, for the young and middle aged women as well as for the future generations.
\end{abstract}

\section{Key words: Cervical cancer, HPV infection, HPV vaccine.}

\section{Introduction}

Cervical cancer is a public health problem. It is broadly accepted that well organized cervical cancer prevention programs based on primary screening with cervical cytology led to impressive reduction in cervical cancer rates in developed countries. However, women in developing countries still suffer a disproportionately high burden of illness from cervical cancer, and it remains the leading cause of cancer death among women. Eighty three percent of the 4,93,000 new cases identified annually in the world occur in developing countries and cervical cancer kills nearly 274,000 women every year ${ }^{1}$.

\section{Causative Agent for Cervical Cancer:}

Human Papilloma Virus (HPV) is a necessary cause of cervical cancer and it has been firmly established biologically and epidemiologically ${ }^{2,3}$. HPV 16 \& 18 account for $70 \%$ of squamous cell carcinoma of cervix ${ }^{4}$. The other HPV responsible for cervical cancer world wide in descending order of frequency are -45, 31, -33, -52, -58 and 35 (IARC) ${ }^{5}$. HPV infection is a sexually transmitted disease having a global prevalence of $10.41 \%$ in women with normal cytology6. Peak level occurs in $20-25 \%$ of women between 20-24 years and then decline. Women $>35$

1. Professor of Gynae and Obs, National Institute of Cancer Research and Hospital,

2. Associate Surgeon, Dept of Obs \& Gynae, SSH

3. Senior Consultant, Dept of Obs \& Gynae, SSH, Dhaka

4. Medical Officer, Dept of Obs \& Gynae, years have persistent HR-HPV types in only cases ${ }^{7,8,9,10}$. The median time to clearance prevalent infection ranges from 4-6 months 1-2 years.

\section{Immune Response in HPV Infection:}

Both innate and adaptive immune response plays role in clearance of HPV infection. In transient HPV infection innate immune response quickly eliminates antigens before they develop memory response ${ }^{12}$. But in persistent HPV infection antibody mediated humoral response accompany cell mediated immune response to mount an effective immune response ${ }^{12}$. Antibody mediated humoral immunity clears free virus particles and prevents reinfection with same HPV type ${ }^{13}$. Cell mediated immune response clears virus infected cells and generates immune memory and lesion regresses ${ }^{14}$.

\section{Risk factors for HPV infection and Neoplasia:}

Risk factors for HPV acquisition, persistence and neoplastic progression are closely linked. Earlier sexual debut and shorter intervals between menarche and sexual debuts is an important risk factor ${ }^{15}$. Cervical immunity, inadequate production of protective cervical mucus and increased cervical ectopy make the young women susceptible to HPV infection ${ }^{15}$. High parity, multiple partners and acquisition of new partners are independent co-factor for cervical carcinogenesis ${ }^{16,17}$. Recently IARC has classified combined oral contraceptives as carcinogenic to cervix and risk is related with increase duration of use $(>10 \mathrm{yrs})^{18}$. Cervical infection with clamydia trachomatis may increase susceptibility to HPV 
infections ${ }^{19}$. Male circumcision has not been linked to female HPV acquisition ${ }^{20}$. Evaluation of relationship between condom use and HPV infection have failed to demonstrate a protective effect of condoms because HPV infections is transmissible through contact with areas of unprotected genital skin ${ }^{21}$.

The association between smoking and HPV persistence is less consistent ${ }^{22-24}$. But IARC has classified tobacco smoking as a cause of cervical cancer $^{25}$. But it is entirely unclear at which stage in cervical carcinogenesis smoking interferes.

\section{HPV-DNA Detection:}

The association of "high risk' HPV type with cervical cancer encouraged people to concentrate their effort in the detection of HPV. US Food \& Drug Administration (FDA) has approved two methodologies for HPV DMA detection ${ }^{5}$. The Hybrid Capture System (HC2) detects 13 HR-HPV types. Since HC2 is less prone to cross-contamination and time and cost to perform that test is minimum, high volume screening using automation is applied to detect HPV DMA in clinical practices. On the other hand Polymerase Chain Reaction (PCR) assay for detection of HPV-DNA have high sensitivity, prone to cross contamination and needs expensive equipment for reading and thus has limited acceptability in clinical applicability. But PCR has the potential to identify HPV types that infect lower genital tract. Rapid HPV tests are now in the market which are affordable and suitable in low resource setting. They are rapid batch test and rapid strip test which had been field-tested in China \& India. Both tests are cheap and less time consuming. The sensitivity of HPV for detection of cervical cancer is superior to that of cytology and specificity is almost equal or less ${ }^{26}$.

\section{Clinical Application of HPV-DNA testing:}

HPV-DNA testing is useful in three clinical application As a triage test to select women showing minor cytological abnormality in pap's smear needing referral for diagnosis and treatment

" As a primary screening test solely or in combination with a pap smear

" As a follow up test for women treated for High Grade Intraepithelial Lesion (HGIL) with local ablative therapy or Loop Electro Excision Procedure (LEEP) to predict cure of treatment

Triage is an additional step interposed between screening and diagnosis to further stratify individuals with positive primary screening results. HPV triage has been shown to be useful in minor cytological lesions like Atypical Squamous Cells of Undetermined Significance (ASCUS) which offers 99\% NPV and in USA HPV testing is included with cytology in primary screening after the age of 30 years and is approved by FDA. Algorithm showing the use of HPV triage in minor cytological

lesions like Atypical Squamous Cells of Undetermined Significance (ASCUS) which offers 99\% NPV and in USA HPV testing is included with cytology in primary screening after the age of 30 years and is approved of FDA.

\section{Algorithm showing the use of HPV triage in minor cytological lesions}

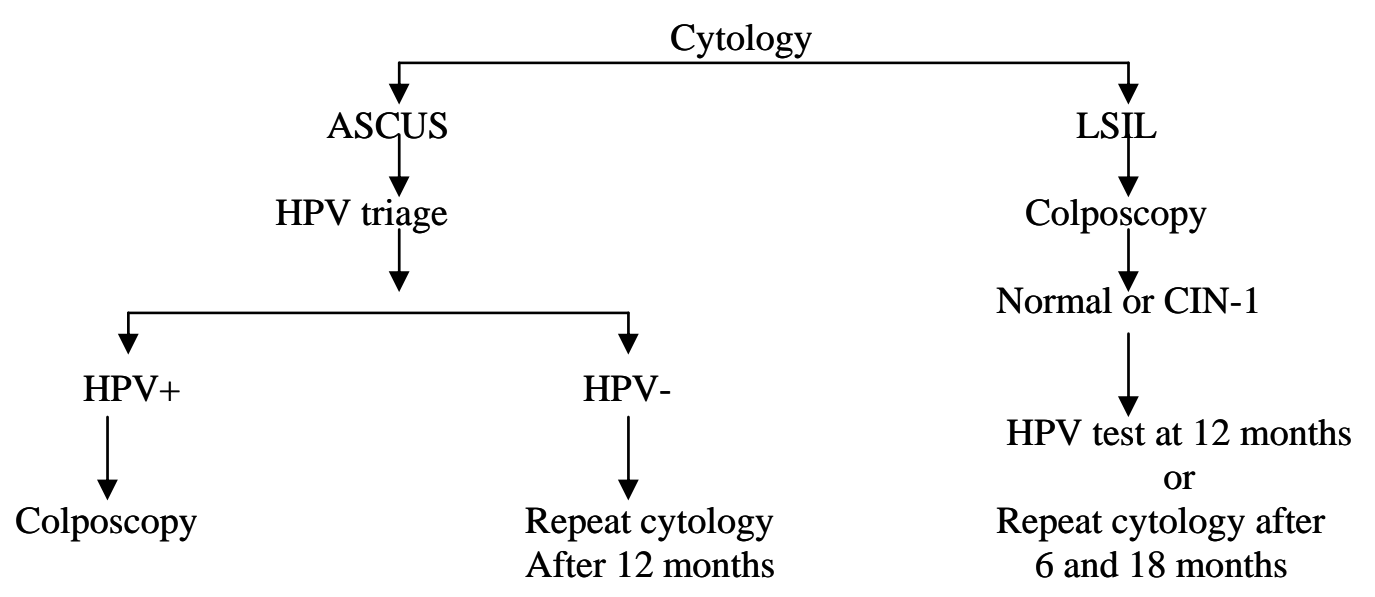

The sensitivity to predict CIN2 and CIN3 within 5 yeas or 10 years is $86 \%$ and $72 \%$ respectively ${ }^{28}$. The NPV of a combined negative test in 5 years is $99.91 \%$.saves $50 \%$ of women from unnecessary colposcopy without compromising sensitivity ${ }^{26,27}$. The use of HPV testing alone in primary screening is being tried. The rationality 


\section{Algorithm showing the use of HPV testing in combination with cytology in primary, screening}

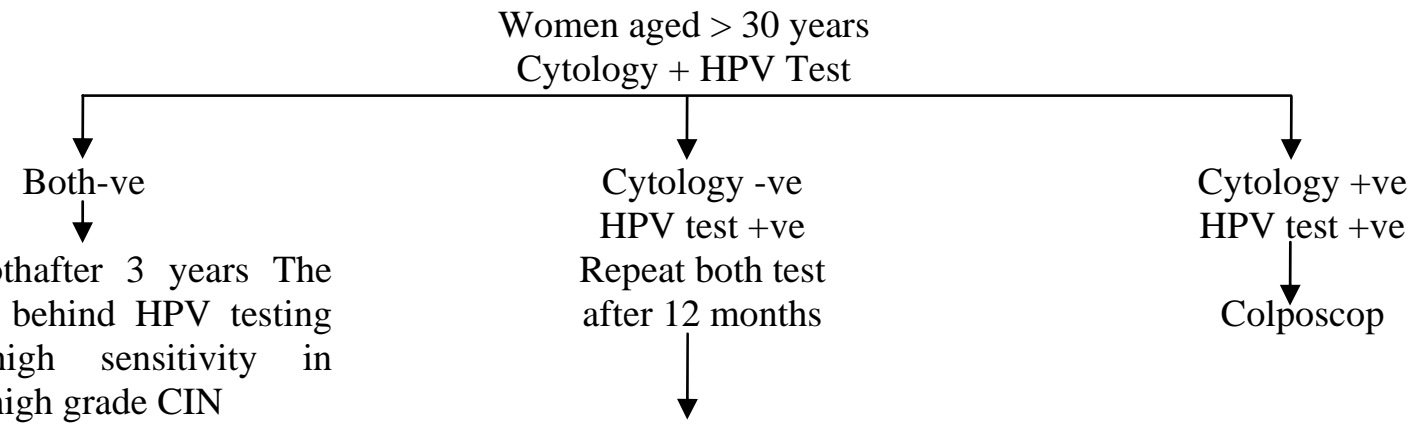

Either test + colposcopy

behind is HPV testis of its high sensitivity in detecting high grade CIN. But it is less specific than cytology due to detection of transient infections that have not produced cytological changes. Thus large pragmatic trials comparing HPV test to cytology alone are needed to assess the impact of primary HPV screening on cancer incidence and mortality.

HPV test has proved its value in follow up of patients treated for CIN. Regular follow up for 2 years detects $10 \%$ treatment failure. HPV testing allows shortening of post treatment surveillance. HPV testing picks up residual disease quicker with higher sensitivity and similar specificity to cytology. Zielinski has proposed combined cytology and HPV testing at 6 and 24 months after treatment and referral back to 5 years cytology screening if all tests are negative ${ }^{29}$.

\section{HPV vaccine:}

Inspite of different approaches in secondary prevention of cervical cancer most women in the world have been denied any form of prevention. Invention of HPV vaccines is a great breakthrough for primary prevention of cervical cancer. The generation of neutralizing antibodies to HPV capsid protein is the rationality for HPV vaccine introduction in the market $^{30,31}$. Two types of vaccines are currently available in the market. Bivalent vaccine is active against HPV-16/18 while Quadrivalent vaccine protects against HPV-16/18/6/11 infections. Both vaccines are given intramuscularly in three shorts over 6 months (0.5 ml - 0, 1-2, 6 months). Since HPV infection is sexually transmitted, immunization to protect against infection and resulting disease must therefore precede the sexual debut. The US Advisory Committee on Immunization Practices (ACIP) Guidelines for HPV vaccination recommends the following issues.

Routine HPV vaccines is recommended for girls between 11-13 years but girls younger than 11 ( $>9$ years) can be vaccinated. Females who have not been infected with any HPV type would receive the full benefit of vaccination.

- The vaccine also offers cross protection against other HPV types like 45 for 18, 31 for 16.

- Both vaccine appear to be generally safe and well tolerated.

- The cut off duration for vaccine protection is still debatable.

- Antibody level falls from peak level after immunization (8-18 months) to plateau level that persists for at least 48 months post vaccination.

Screening needs to be continued simultaneously with vaccination for foreseeable future because only vaccination of primary target population will achieve low coverage. Also vaccination will not protect women who are infected with HPV types which are not included in the vaccine. Evaluating the impact of HPV vaccine and screening on cervical cancer on mathematical models it has been shown that .vaccination alone, assuming $70 \%$ coverage, reduces cervical cancer incidence by $48 \%$. Vaccination plus screening three times per lifetime (begnining between ages 30 and 35 and repeated at 5 years intervals) reduces overall cervical cancer incidence by $66 \%{ }^{32}$.

The registration of first generation of HPV vaccines represents a highly significant milestone in current efforts to reduce the impact of cervical cancer globally. We now have the capacity to prevent cervical cancer not only through secondary prevention by screening and treatment of CIN 2/3 but also through primary prevention. Careful integration of primary and secondary prevention programs should lead to greater reductions in cervical cancer, 
ervical cancer.

\section{Reference}

1. Ferlay J, Bray F, Pisani P, Parkin DM. GLOBOCAN 2002 cancer incidence, Mortality and prevalence worldwide. IARC Cancer Base No.5 version 2.0 Lyon: IARC Press; 2004.

2. Bosch FX, Manos MM, Munoz N, Shernan M, Jaysen A, Peto J et al. The IBSCC study group. Prevalence of Human Papillomavirus in cervical cancer; a worldwide perspective, J Natl cancer inst 1995; 87: 796-802.

3. Walboomers TM, Jacobs MV, Manos MM, Bosch Fx, Kummer JA, Shah KV et al. Human Papilloma virus is a necessary cause of invasive cervical cancer worldwide. J Pathol 1999; 189: 12-9.

4. Munoz N, Bosch Fx, Castellsague X, Diaz M, De Sanjose S, Hammouda $D$ et al. Against which human papillomavirus type shall we vaccinate and screen? The international perspective. Int $\mathrm{J}$ Cancer 2004; 41: 278-85.

5. IARC Monographs on the evaluation of carcinogenic risks to humans. Human Papillomavirus, Vol.90. Lyon: International Agency for Research on Cacner, In press.

6. de Sanjose S, La investigacon sobre la infection porvirus del papiloma humans y el cancer de cuello uterino en Espana. IN: El virus del papilloma humons y cancer: Epidemiologia y Prevencon. Ed: S. de Sanjose \&A. Garcia, EMISA, Madrid, 2006, in press.

7. Melkert PWJ et al. Int. J Cancer 1993; 53: 919-923.

8. Boshart M et al. EMBO J 1984; 3: 1151-1157.

9. Meijer CJLM et al. In Munoz N. Mosch Fx. Shah KV (eds): The Epidemiology of Human Papillomavirus and cervival cancer. Lyon, IARC scientific Publication, 1992. pp 27-28.

10. Rohan J et al. Int J Cancer 1991, 49: 856-860

11. Trottier H, France EL. The Epidemiology of genital human papillomavirus infection. Vaccine 2006; 24 (suppl I): S4-S15

12. Konya J, Dillner J. Immunity to oncogenic human papillomavirus. Vaccine 2005; Epub ahead of print.

13. de Jonj A, Van Poelgeest Ml. Van der Hulst JM, Drijshout JW, Flenren GJ, Melief CJ et al. Human Papillomavirus type 16positive cervical cancer is associated with impaired CD4 T cell immunity agaisnt early antigens E2 and E6. Cancer Res 2004: 64(15); 5449-55.

14. Stanley M. Immune responses to human Papillomavirus. Vaccine 2005; Epub ahead of print.

15. Kahn JA. Rosenthal SL, Suecop PA, HOGY, Burk RD. The interval between menarche and age of first sexual intercourse as a risk factor for subsequent HPV infection in adolescent and young adult women. J Paediatr. 2002; 141(5): 718-23.

16. Cervical carcinoma and reproductive factors: collaborative reanalysis of individual data on 16,563 women with cervical cancer and 33,542 women without cervical cancer from 25 epidemiological studies: Int J Cancer 2006: 119: 1108-24.

17. Winer RL; Lee Sk, Hughes JP. Adam DE. Kiviat NB. Koutsky LA. Genital human papillomarivus infection: incidence and risk factor in a cohort of female university students. Am J Epidemiol. 2003; 157(3): 218-26.

18. Cogliono V. Grosse Y, Baan R, Staif K, Seretan B, EL Ghissassi F. Carcinogenecity of combined oestrogen progestogen contraceptives and menopausal treatment. Lancet Oncol 2005; 6: 552-3.
19. Samoff E, Koumaus EH. Markowitz LE, Sternberg M, Sawyer MK, Swass D et al. Association of clamydia trachomatis with persistence of high risk type of HPV in a cohort of female adolescents. Am J Epidemiol 2005; 162(7): 668-75.

20. Winer RL, Koutsky LA. The epidemiology of HPV infections: In: Rohan J. Shank. Editors. Cervical Cancer: From etiology to prevention. Dordrecht. The Netherlands: Kluwer Academic Publishers: 2004. P.143-87.

21. Manhart LE, Koustky LA. Do condoms prevent genital HPV infections, external genital warts or cervical neoplasia. A meta analysis. Sex Tsansm Dis 2002; 29)4): 725-35.

22. HOGY. Bierman R. Beardsley L, Chang Cl. Burk RD. Natural history of cervicovaginal papillomavirus infection is young women. $\mathrm{N}$ Engl J Med 1998: 338(7): 423-8.

23. Silins I. Ryd W, Strand A. Wadell G, Tornberg S, Hansson BG et al. Clamydia trachomatis infectm and persistence of human papillomarivus. Int J Cancer 2005: 116(1): 110-5.

24. Richardson H, Abrahamowicz M. Tellier PP. Kelsall G Du BR Ferenczy A et al. Modifiable risk factors assoicated with clearance of type specific cervical human papillomavirus infections in a cohort of university students. Cancer Epidemiol Biomarkers Prev 2005; 14(5): 1149-56

25. IARC TEchnical reports N3. Tobacco smoke and involuntary smoking. IARC press, 2004.

26. Solomon D. Schiffman M \& Tarone. R (2001) Comparison of three management strategies for patients with ASCUS: Baseline results from a randomized trial J. Natl Cancer Inst. 93. 293-299.

27. Manos MM, Kinney Wk. Hurley L.B. Sherman M.E et al. Identifying women with cervical neoplasia: using HPV DNA testing for equivocal papanicolaou results. JAMA.1999. 281 1605-1610.

28. Sherman ME. Lovinez AT. Scott DR. Wachoider S, Castle PE, Glass AG et al. Baseline cytology. HPV testing \& risk for cervical neoplasia: a 10 year cohort analysis. J Natl. cancer Inst 2003; 95(1): 46-52.

29. Zielinski GD. Bais AG. Helmershost TJ. Verheijen RH." de schipper FA, Smjders PJ et al. HPV testing and monitoring of women after treatment of CIN 3: review of literature and meta analysis. Obstet Gynaecol Surv 2004; 59(7): 543-53

30. Herper DM. Franco EL, Wheeler CM. Moscicki AB Romanowsk 8 et al. Sustained efficacy upto 4.5 years of a bivalent L1 VLP vaccine against HPV type 16 \& 18: follow up from a randomized control trial.Lancet 2006: 367(9518): 1247-55.

31. MaoC, Koutsky LA. Ault KA, Wheeler CM. Brown DR. Wiley DJ et al. Efficacy of HPV -16 vaccine to prevent CIN a randomized controlled trial. Obstet Gynaecol 2006: 107(1): 18-27

32. Garnett GP. Kim JJ, French K et al. Modelling the impact of HPV vaccines on cervical cancer and screening programmes Vaccine 